VOL. 7 (1972), 101-104.

\title{
On complemented chief factors of finite soluble groups
}

\section{D.W. Barnes}

Let $G=H_{0}>H_{1}>\ldots>H_{r}=1$ and $G=K_{0}>K_{1}>\ldots>K_{r}=1$ be two chief series of the finite soluble group $G$. Suppose $M_{i}$ complements $H_{i} / H_{i+1}$. Then $M_{i}$ also complements precisely one factor $k_{j} / K_{j+1}$ of the second series, and this $k_{j} / K_{j+1}$ is $G$-isomorphic to $H_{i} / H_{i+1}$. It is shown that complements $M_{i}$ can be chosen for the complemented factors $H_{i} / H_{i+1}$ of the first series in such a way that distinct $M_{i}$ complement distinct factors of the second series, thus establishing a one-to-one correspondence between the complemented factors of the two series. It is also shown that there is a one-to-one correspondence between the factors of the two series (but not in general constructible in the above manner), such that corresponding factors are $G$-isomorphic and have the same number of complements.

Throughout this note, $G$ is a finite soluble group. Let $A$ be an irreducible $G$-module which occurs as a complemented chief factor of $G$, and let $C=C_{G}(A)$. Let $R=R(A)$ be the intersection of all normal subgroups $D$ of $G$ such that $D<C$ and $C / D$ is isomorphic to $A$ (as $G$-module). Clearly $C / R$ is isomorphic to the direct sum of $d$ copies of $A$ for some $d$. In [3], Gaschütz proves that the number of complemented factors isomorphic to $A$ in a chief series of $G$ is $d$. This follows at

Received 6 March 1972. This note elaborates arguments of Gaschütz; see [2, Satz 3], [3, Satz 4.1], and also Carter, Fischer, and Hawkes [1, Lemma 2.6]. 
once from

LEMMA 1. Let $H / K$ be a chief factor of $G$. Then $H / K$ is complemented and isomorphic to $A$ if and only if $C \geq H R>K R$.

Proof. Suppose $H / K \simeq A$ and let $M$ be a complement to $H / K$ in $G$. Then $H \cap \operatorname{Core}_{G}(M)=K$ and $H$. Core $G_{G}(M)=C_{G}(H / K)=C$. Thus $C \geq H R \geq K R, \quad C /$ Core $_{G}(M) \simeq H / K \simeq A$, and $\operatorname{Core}_{G}(M) \geq R$. If $K R=H R$, then

$$
\operatorname{Core}_{G}(M)=K R \cdot \operatorname{Core}_{G}(M)=H \cdot \operatorname{Core}_{G}(M)=C \text {, }
$$

and we have $K=H \cap$ Core $_{G}(M)=H$. Therefore $H R>K R$.

Suppose conversely that $C \geq H R>K R$. Then $H / K \cong H R / K R$ which, being a chief factor of $G$ between $C$ and $R$, is isomorphic to $A$. Since $C / R$ is a completely reducible module, there exists a maximal submodule $D / R$ of $C / R$ such that $D \cap H R=K R$. Since $C_{G / D}(C / D)=C / D$, $G / D$ splits over $C / D$ and all complements to $C / D$ in $G / D$ are conjugate. If $M / D$ is a complement to $C / D$, then $M$ complements $H / K$. Thus $H / K$ is complemented.

We have seen that $H / K$ has one conjugacy class of complements for each maximal submodule $D / K R$ of $C / K R$ not containing $H R / K R$. We now determine explicitly the number of these.

LEMMA 2. Let $A$ be an irreducible G-module and let $F$ be the endomorphism ring of $A$. Let $V$ be the direct sum of $d$ copies of $A$ and let $H$ be a minimal submodule of $V$. Then the number of maximal submodules of $V$, not containing $H$ is $|F|^{d-1}$.

Proof. By Schur's Lemma, $F$ is a field (commutative since finite). $V=\left\{\left(a_{1}, \ldots, a_{d}\right\} \mid a_{i} \in A\right\}$ and we have maps $\varepsilon_{i}: A \rightarrow V$ defined by $\varepsilon_{i}(a)=(0,0, \ldots, a, 0, \ldots)$ where the non-zero entry is in the $i$ th place. Let $M$ be any maximal submodule of $V$. Then there is a homomorphism $\alpha: V+A$ with $\operatorname{ker} \alpha=M$. For each $i$, we have $\lambda_{i} \in F$ defined by $\lambda_{i}=\alpha \varepsilon_{i}: A \rightarrow A$, and $\left(a_{1}, \ldots, a_{d}\right) \in M$ if and only if 
$\sum_{i=1}^{d} \lambda_{i}\left(a_{i}\right)=0$. Conversely, for any $\lambda_{1}, \ldots, \lambda_{d} \in F$, not all zero, putting $\alpha\left(a_{1}, \ldots, a_{d}\right)=\sum_{i=1}^{d} \lambda_{i}\left(a_{i}\right)$ defines an epimorphism $\alpha: V \rightarrow A$ whose kernel is a maximal submodule of $V$. For $\lambda \in F, \lambda \neq 0$, $\left(\lambda \lambda_{1}, \ldots, \lambda \lambda_{d}\right)$ defines the same maximal submodule as $\left(\lambda_{1}, \ldots, \lambda_{d}\right)$. Thus the number of maximal submodules is $\frac{q^{d}-1}{q-1}$, where $q=|F|$. The number containing $H$ is $\frac{q^{d-1}-1}{q-1}$ and the result follows.

Since the number of complements in a conjugacy class is $|A|$ provided $C \neq G$, by pairing complemented factors $H_{i} / H_{i+1}$ and $K_{j} / K_{j+1}$ isomorphic to $A$, for which $H_{i} R / R$ and $K_{j} R / R$ appear at the same level in the lattice of submodules of $C / R$, we have

THEOREM 1. Let $G=H_{0}>H_{1}>\ldots>H_{r}=1$ and $G=K_{0}>K_{1}>\ldots>K_{r}=1$ be two chief series of the finite soluble group $G$. Then there exists a one-to-one correspondence between the factors of the two series, such that corresponding factors are G-isomorphic and have the scome number of complements.

We now prove

THEOREM 2. Let $G=H_{0}>H_{1}>\ldots>H_{r}=1$ and $G=K_{0}>K_{1}>\ldots>K_{r}=1$ be two chief series of the finite soluble group $G$. Then complements $M_{i}$ can be chosen for the complemented factors $H_{i} / H_{i+1}$ of the first series, in such a way that distinct $M_{i}$ complement distinct factors of the second series.

By the discussion above, it is sufficient to prove

LEMMA 3. Let $V=U_{0}>U_{1}>\ldots>U_{d}=0$ and $V=V_{0}>V_{1}>\ldots>V_{d}=0$ be composition series of the completely reducible module $V$. Then there exist maximal submodules $W_{1}, W_{2}, \ldots, W_{d}$ of $V$ such that 


$$
u_{i}=w_{1} \cap w_{2} \cap \ldots \cap w_{i} \text { and } v_{i}=w_{\alpha_{1}} \cap w_{\alpha_{2}} \cap \ldots \cap w_{\alpha_{i}}
$$

for some permutation $\alpha_{1}, \ldots, \alpha_{d}$ of $1,2, \ldots, d$.

Proof. The result clearly holds for $d=1$. We use induction over d. For some $k, v_{k} \neq U_{1}$ but $v_{k+1} \leq U_{1}$. By the complete reducibility, there exists a minimal submodule $z$ such that $V_{k}=z+V_{k+1}$. By induction, there exist maximal submodules $T_{2}, \ldots, T_{d}$ of $U_{1}$ such that $U_{i}=T_{2} \cap \ldots \cap T_{i}$ and $V_{i} \cap U_{1}=T_{B_{2}} \cap \ldots \cap T_{\beta_{j}}$ for some $\beta_{2}, \ldots, \beta_{j}$. Put $w_{1}=U_{1}, w_{i}=T_{i}+2(i=2, \ldots, d)$. Since $V / Z=U_{1}$ and $T_{i}=W_{i} \cap U_{1}$, these $W_{1}, \ldots, W_{d}$ satisfy the requirements.

\section{References}

[1] Roger Carter, Bernd Fischer, Trevor Hawkes, "Extreme classes of finite soluble groups", J. Algebra 9 (1968), 285-313.

[2] Wolfgang Gaschütz, "Die Eulersche Funktion endlicher auflösbarer Gruppen", IZZinois J. Math. 3 (1959), 469-476.

[3] Wolfgang Gaschütz, "Praefrattinigruppen", Arch. Math. 13 (1962), $418-426$.

Department of Pure Mathematics, University of Sydney, Sydney, New South Wales. 